\title{
EVALUATION OF MECHANICAL PROPERTIES OF MAXILLOFACIAL SILICONE AFTER LONG TERM EXPOSURE TO DIFFERENT CONDITIONS
}

\author{
Hebatalla Mahmoud El Afandy* and Aya Mohamed fawzy *
}

\begin{abstract}
Purpose: The aim of this study was to examine the effect of dark room storage, neutral soap (shampoo) and outdoor weathering on the mechanical properties (roughness, flexibility and elastic recovery) of maxillofacial silicone elastomer.
\end{abstract}

Material and Methods: 60 specimen were prepared and divided into 3 groups $(n=20)$.And conditioned differently as follows: Group I: Dark room storage; Group II: Neutral soap disinfection (shampoo); Group III: Outdoor natural weathering for 2 months. Specimens were subjected to roughness evaluation using scanning electron microscope, flexibility test and elastic recovery by means of materials testing device (Model LRX-plus; Lloyd Instruments Ltd., Fareham, UK) before storage, after 1 month and one more after another 1 month.

Results: There was significant increase in the roughness of natural weathering group, roughness decreases in dark room and shampoo groups. There was increase also in the flexibility in natural weathering group and decrease in dark room and shampoo groups. Finally in the recovery percentage there was decrease in natural weathering group but increase in both dark room and shampoo groups.

Conclusions: Natural weathering conditions affected the mechanical properties of maxillofacial silicone.

Key words: Maxillofacial silicone elastomer; Roughness; Mechanical properties

\section{INTRODUCTION}

Several maxillofacial materials are used to interchange missing facial parts which have been missed through trauma or disease. Silicone elastomers is the furthermost commonly used material to construct maxillofacial prostheses for the reason of its texture, strength, durability's and simplicity in handling, coloring, and patient, comfort. There are many beneficial properties of silicone prosthetics that dedicate silicone as the most appropriate material for facial prostheses such as good biocompatibility and biodurability, wide-ranging serviceability temperature range,

\footnotetext{
* Lecturer of prosthodontics, Faculty of oral and Dental medicine, Future university. Cairo, Egypt.
} 
non-adhesive properties, low toxicity, possible optical transparency, slight chemical reactivity, and brilliant resistance to attack by oxygen, ozone, and daylight. The physical and mechanical properties of silicone elastomer are dependent on the degree of cross-linking, the kind and concentration of fillers in the elastomer network. The degree of crosslinking, depends on the nature and concentration of the thermal initiator, the fillers, the additives, and cure temperature and polymerization time. ${ }^{1}$

Silicon are long chain molecules collected of alternating chain of silicone and oxygen atoms, by altering the length of this silicon-oxygen chain silicones can be formed in the form of fluids, resins, or elastomers (rubbers). And accordingly acquire improved physical and chemical properties. The unexpected properties of silicones are due to the special features of the silicon-oxygen bonds in their backbone. Because the silicon-oxygen bond is much tougher than the carbon-carbon bond of organic polymers, silicones make improved electric insulators and are more resistant to oxidation. Chemically they are named as polydimethyl siloxane (PDMS) and classified into main types based on vulcanizing temperature: Room temperature vulcanizing (RTV) silicones and high-temperature vulcanized (HTV) silicones. ${ }^{2}$

Primary factors of weathering including ultraviolet (UV) rays, temperature, moisture, and secondary factors such as deposition of microscopic deposits in the porosities of the material's surface and use of disinfecting means, contributed to the maxillofacial material instability. Physical properties such as hardness, surface roughness, and tensile strength were also evaluated extensively. The wear time of facial silicones exposed to environment averages from 3 months to 1 year of use. ${ }^{3,4,5}$

Preceding studies have discovered a varied diversity of testing approaches and conditions used to survey the mechanical and physical properties of poly dimethylsiloxane maxillofacial materials. The tensile strength of the silicone elastomer offers an general strength of the material and the resultant elongation provides a sign of the flexibility of the prosthesis. Prosthesis with a high elongation at break is required mainly when peeling a nasal or eye prostheses from facial tissue. The hardness of the maxillofacial material is similarly a degree of flexibility and is vital since it is necessary to have a material with comparable hardness to the lost facial tissue. $^{6}$

Roughness is the measure of the finer irregularities of surface texture that are characteristic in the materials. Surface roughness average $(\mathrm{Ra})$ is estimated as the arithmetic regular deviation of the surface valleys and peaks conveyed in micro-inches or micrometers. If these deviations are great, the surface is rough; if they are minor, the surface is smooth. For improved investigation of the surface of the specimens, Scanning Electron Microscopy (SEM) was performed. ${ }^{1}$

The tear resistance of maxillofacial materials is a composite phenomenon and reliant on on the form of the test specimens and also on the rapidity of testing (crosshead speed). The greater the rate of stressing the less time the molecules have to reorganize the stress and this leads early tearing of the specimen therefore representing a low rate for tear strength. Consequently data on tear strength of maxillofacial materials has to be cautiously interpreted. ${ }^{6}$

The conditioning stages were designated to mimic silicone prosthesis in service for 18-24 months. Considering that each day patients wear their prosthesis for $8-12 \mathrm{~h}$, through which it is expected to be exposed at least $6 \mathrm{~h}$ of daylight, typical environmental conditions and perspiration, whereas the prosthesis is on the defect place. In addition, before sleeping, patients spend a regular of 5 min washing their prostheses. Therefore, 1 month of service equals $180 \mathrm{~h}$ of daylight aging and 150 min of storage in cleansing solutions. ${ }^{7}$

Earlier studies approve that extra developments in the mechanical and physical properties are essential 
for poly dimethylsiloxane maxillofacial materials in specific the thin margin tear strength, flexibility, roughness and recovery percentage. Consequently the key goal of this study was to analyze a variety of clinically essential properties for the commercially existing maxillofacial materials in order to aid in evaluating of a novel maxillofacial prosthetic material:

\section{MATERIAL AND METHODS}

Room temperature silicone* used to produce soft prosthesis. The material is based on additionlinking vinyl polydimethylsiloxanes. Apply the silicone either using the mixing cannula or directly from the cartridge onto a suitable pad (glass plate, coated paper, etc.) into the molds to obtain the specimens. The material is completely hardened without heating after 12 hours.

A total of 60 specimen were prepared and divided into three groups $(n=20)$. And conditioned differently as follows: Group I: the specimens were stored in dark at room temperature $\left(23 \pm 2^{\circ} \mathrm{C}\right)$, Group II: Neutral soap disinfection (shampoo) ${ }^{* *}$, which is carried on and Group III: Outdoor natural weathering. All the specimens stored for 2 months. The measurements were taken before storage, after 1 month then after the other 1 month (follow up period).

\section{Measurement of Roughness:}

The optical means tend to achieve the essential measureable description of surface topography without contact. ${ }^{8}$

Specimens were photographed by means of USB Digital microscope with a built-in camera*** linked with an IBM compatible personal computer by means of a fixed magnification of $120 \mathrm{X}$. Images were documented with a resolution of $1280 \times 1024$ pixels per image. Digital microscope images were cropped to 350 x 400 pixels by means of Microsoft office picture manager to specify/standardize area of roughness measurement. The images were examined by WSxM software. ${ }^{* * * *} 9$

The WSxM software, all limits, sizes, frames and measured parameters are conveyed in pixels. Consequently, system standardization was made to change the pixels into absolute real world units. Standardization was prepared by matching an object of known size (a ruler in this study) with a scale generated by the software.

Consequently, a 3D image of the surface profile of the specimens was produced. Five 3D images were gathered for each specimen, in the central area and in the sides at area of $10 \mu \mathrm{m} \times 10 \mu \mathrm{m}$

WSxM software was used to estimate regular of heights ( $\mathrm{Ra}$ ) stated in $\mu \mathrm{m}$, which can be presumed as a reliable indices of surface roughness. ${ }^{10}$

\section{Measurement of flexibility:}

The tests were done using NEXYGEN from Lloyd Devices.

Test procedure according to ANSI/ADA specification No. 18

Samples were subjected to flexibility test using materials testing device (Model LRX-plus; Lloyd Instruments Ltd., Fareham, UK) with a load cell of $500 \mathrm{~N}$ and data were documented using computer software (Nexygen-MT Lloyd Instruments). An initial compressive load 125 gr was applied through materials testing device at a crosshead speed of 5 $\mathrm{mm} / \mathrm{min}$. then the height was recorded in $(\mathrm{mm})$ as

\footnotetext{
* Multisil-Epithetik, bredent GmbH \& Co.KG e-mail: R.D@bredent.com.

** Nefertari Hair shampoo, Egypt,www.nefertaribodycare.com.

*** (Scope Capture Digital Microscope, Guangdong, China)

**** (Ver 5 develop 4.1, Nanotec, Electronica, SL)
} 
reading $(\boldsymbol{a})$. Within $10 \mathrm{sec}$. Final load of $1250 \mathrm{gr}$ was applied and the height was recorded in $(\mathrm{mm})$ again as reading $(\boldsymbol{b})$.

\section{Flexibility calculation;}

- Flexibility as \% was calculated using following equation;

Flexibility $=[(\boldsymbol{a}-\boldsymbol{b}) /$ sample height $] * 100$

\section{Measurement of Recovery:}

The tests were done using NEXYGEN from Lloyd Devices.

\section{Test procedure according to ANSI/ADA specifica- tion No. 18}

The upper plate of machine was lowered till it touch the sample, then the height was recorded in $(\mathrm{mm})$ as reading $(\boldsymbol{a})$. Sample was deformed to height of $16 \mathrm{~mm}$ for $5 \mathrm{sec}$. then the deforming force released. The upper plate of machine was lowered again till it touch the sample, then the height was recorded in $(\mathrm{mm})$ as reading $(\boldsymbol{b})$.

\section{Recovery calculation;}

- Recovery as \% was calculated using following equation;

\section{Recovery $=[1-(\boldsymbol{a}-\boldsymbol{b}) /$ sample height $] * 100$}

\section{RESULTS:}

Data were presented as means and standard deviation (SD) values. In this laboratory study the comparison between the three groups was done by means of one-way ANOVA followed by Tukey`s post-hoc test for numerous comparisons between each two groups. While comparison between follow up periods in the same group were performed by paired T-test. Statistical analysis was performed with SPSS ${ }^{*} 22^{\circledR}$ and Microsoft Excel ${ }^{* *} 2016$ The significant level was set at $\mathrm{P} \leq 0.05$.

\section{Flexibility Percentage:}

The results of the flexibility percentage of the tested material were listed in table (1) and showed in figure $(1,2)$. Mean and standard deviation values of flexibility percentage after 1 month were $(5.06 \pm$ $0.46),(4.97 \pm 0.29)$ and $(3.92 \pm 1.31)$, while 2 months were $(4.39 \pm 1.14),(4.49 \pm 0.31)$ and $(6.97 \pm 1.53)$ for dark room, shampoo and natural weathering groups respectively.

One-way ANOVA (analysis of variance) test shown that there was significant difference among all groups $(P=0.000)$. Further statistical analysis was performed using Tukey post hoc test which revealed insignificant difference in means with the same superscript letters $\mathrm{P}>0.05$, while revealed significant difference in means with different superscript letters as $p<0.05$. Paired t test was used to relate among two successive follow up period in each group and revealed significant difference in all groups as $\mathrm{p}<0.05$

TABLE (1): The flexibility percentage of the tested groups:

\begin{tabular}{|c|c|c|c|c|c|}
\hline & \multicolumn{2}{|l|}{ After 1 month } & \multicolumn{2}{c|}{$\begin{array}{c}\text { After 2 } \\
\text { months }\end{array}$} & \multirow{2}{*}{ P value } \\
\hline & $\begin{array}{c}\text { Mean } \\
\%\end{array}$ & SD & $\begin{array}{c}\text { Mean } \\
\%\end{array}$ & SD & \\
\hline Dark room & $5.06^{\mathrm{a}}$ & 0.46 & $4.39^{\mathrm{a}}$ & 1.14 & $0.04^{*}$ \\
\hline Shampoo & $4.97^{\mathrm{a}}$ & 0.29 & $4.49^{\mathrm{a}}$ & 0.31 & $0.03^{*}$ \\
\hline $\begin{array}{c}\text { Natural } \\
\text { weather }\end{array}$ & $3.92^{\mathrm{b}}$ & 1.31 & $6.97^{\mathrm{b}}$ & 1.53 & $0.004^{*}$ \\
\hline P value & $0.000^{*}$ & $0.000^{*}$ & \\
\hline
\end{tabular}

M\%; Mean Percentage, SD; Standard Deviation, P; Probability level Means with same superscript letter in the same column were insignificant different

Means with different superscript letter the same column were significant different *significant difference
* Statistical Package for Social Science, IBM, USA.

** Microsoft Co-operation, USA. 


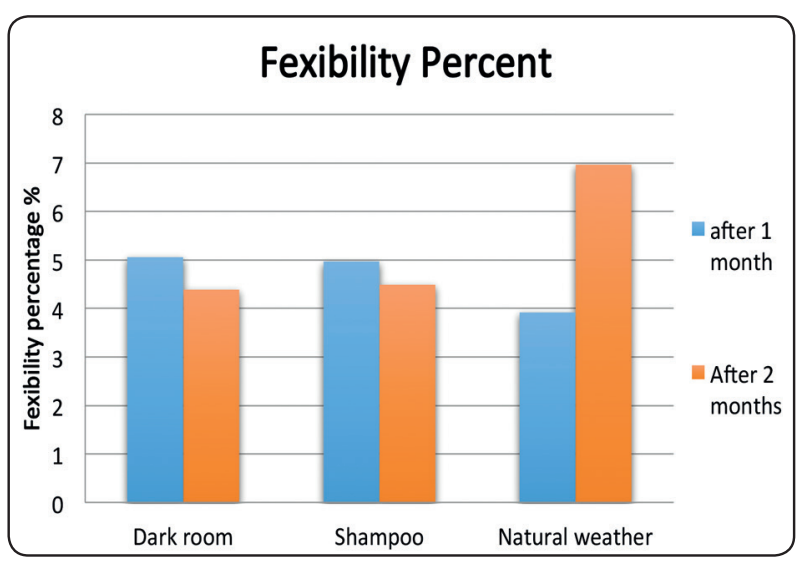

Fig. (1): Bar chart represents flexibility percentage in tested groups.

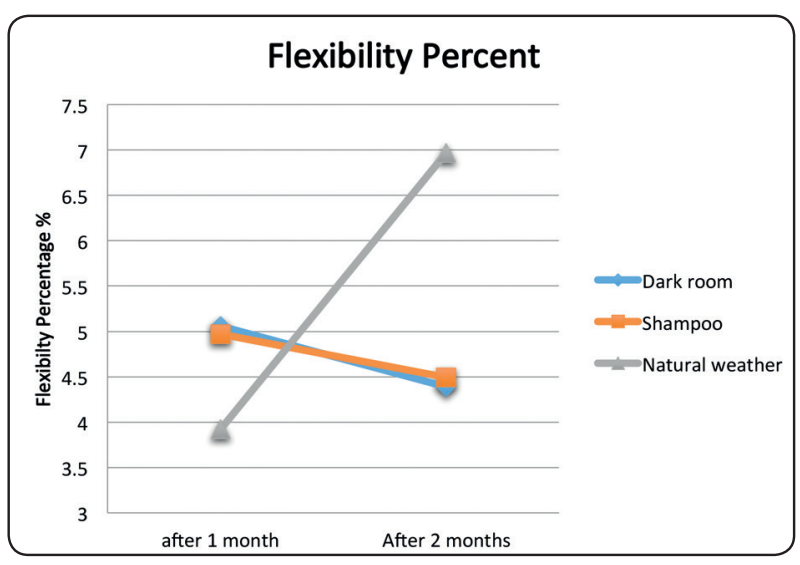

Fig. (2): Line chart represents changes in flexibility percentages of tested groups during follow up

\section{Recovery Percentage:}

The results of the recovery percentage of the tested material were listed in table (2) and showed in figure $(3,4)$. Mean and standard deviation values of recovery percentage after 1 month were $(94.46 \pm$ $0.64),(93.37 \pm 0.14)$ and $(94.87 \pm 0.21)$, while after 2 months were $(95.6 \pm 1.1),(95.5 \pm 0.31)$ and $(93.02 \pm$ 1.6) for dark room, shampoo and natural weathering respectively.

One-way ANOVA (analysis of variance) test revealed that there was significant difference among all groups $(P=0.000)$. Further statistical analysis was performed using Tukey post hoc test which revealed insignificant difference in means with the same superscript letters $\mathrm{P}>0.05$, while revealed significant difference in means with different superscript letters as $p<0.05$. Paired t test was used to compare among two follow up periods for each group and revealed insignificant difference (P> 0.05 ) in dark room \& Natural weathering groups, while revealed significant difference $(\mathrm{P}<0.05)$ in Shampoo group.

TABLE (2): The recovery percentage of the tested groups:

\begin{tabular}{|c|c|c|c|c|c|}
\hline & \multicolumn{2}{|c|}{ After 1 month } & \multicolumn{2}{|c|}{ After 2 months } & \multirow{2}{*}{ P value } \\
\hline & Mean \% & $\mathrm{SD}$ & Mean \% & SD & \\
\hline $\begin{array}{l}\text { Dark } \\
\text { room }\end{array}$ & $94.46^{\mathrm{a}}$ & 0.64 & $95.61^{\text {a }}$ & 1.15 & 0.06 \\
\hline Shampoo & $93.37^{\mathrm{b}}$ & 0.14 & $95.50^{\mathrm{a}}$ & 0.31 & $0.01 *$ \\
\hline $\begin{array}{l}\text { Natural } \\
\text { weather }\end{array}$ & $94.87^{c}$ & 0.21 & $93.02^{b}$ & 1.60 & 0.1 \\
\hline P value & \multicolumn{2}{|c|}{$0.000 *$} & \multicolumn{2}{|c|}{$0.000 *$} & \\
\hline
\end{tabular}

M\%; Mean Percentage, SD; Standard Deviation, P; Probability level

Means with different superscript letter the same column were significant different

*significant difference

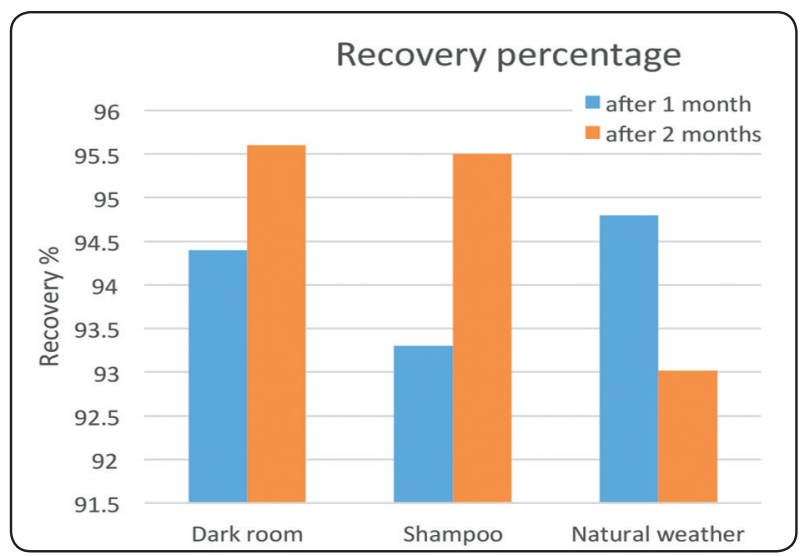

Fig. (3): Bar chart represents recovery percentage in tested groups. 


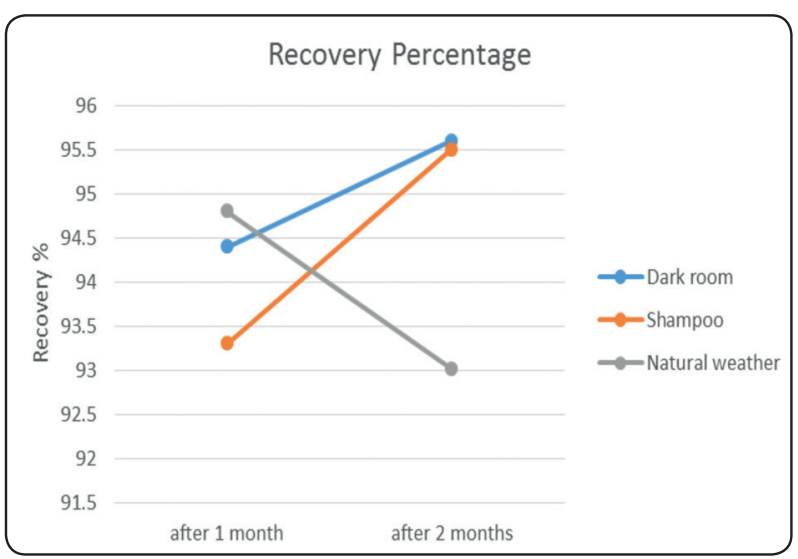

Fig. (4): Line chart represents changes in recovery percentages of tested groups during follow up

\section{Roughness}

The results of the roughness of the tested material were listed in table $(3)$ and showed in figure $(5,6)$. Mean and standard deviation values of roughness after 1month were $(0.266 \pm 0.01),(0.261 \pm 0.003)$ and $(0.253 \pm 0.004)$, while after 2 months were $(0.257 \pm 0.004),(0.255 \pm 0.001)$ and $(0.261 \pm 0.001)$ for dark room, shampoo and natural weathering groups respectively.

One-way ANOVA test revealed that there was significant difference among all groups $(\mathrm{P}=$ $0.000)$. Further statistical analysis was performed using Tukey post hoc test which revealed that there was significant difference in means with different superscript letters $(\mathrm{P}<0.05)$, while revealed insignificant difference in means with the same superscript letters $(\mathrm{P}>0.05)$ Paired t test was used to compare among two follow up periods in each group and revealed insignificant difference in control \& shampoo groups $(\mathrm{P}>0.05) \mathrm{m}$ while revealed significant difference in natural weathering group $(\mathrm{P}<0.05)$.
TABLE (3): The roughness of the tested groups:

\begin{tabular}{|c|c|c|c|c|c|}
\hline & \multicolumn{2}{|c|}{ After 1 month } & \multicolumn{2}{c|}{ After 2 months } & \multirow{2}{*}{ P value } \\
\hline & Mean & SD & Mean & SD & \\
\hline Dark room & $0.266^{\mathrm{a}}$ & 0.01 & $0.257^{\mathrm{a}}$ & 0.0045 & 0.5 \\
\hline Shampoo & $0.261^{\mathrm{b}}$ & 0.003 & $0.255^{\mathrm{b}}$ & 0.0018 & 0.7 \\
\hline $\begin{array}{c}\text { Natural } \\
\text { weather }\end{array}$ & $0.253^{\mathrm{c}}$ & 0.004 & $0.261^{\mathrm{c}}$ & 0.0012 & $0.001^{*}$ \\
\hline P value & \multicolumn{2}{|c|}{$0.000^{*}$} & \multicolumn{2}{|c|}{$0.000^{*}$} & \\
\hline
\end{tabular}

M; Mean, SD; Standard Deviation, P; Probability level

Means with different superscript letter the same column were significant different

*significant difference

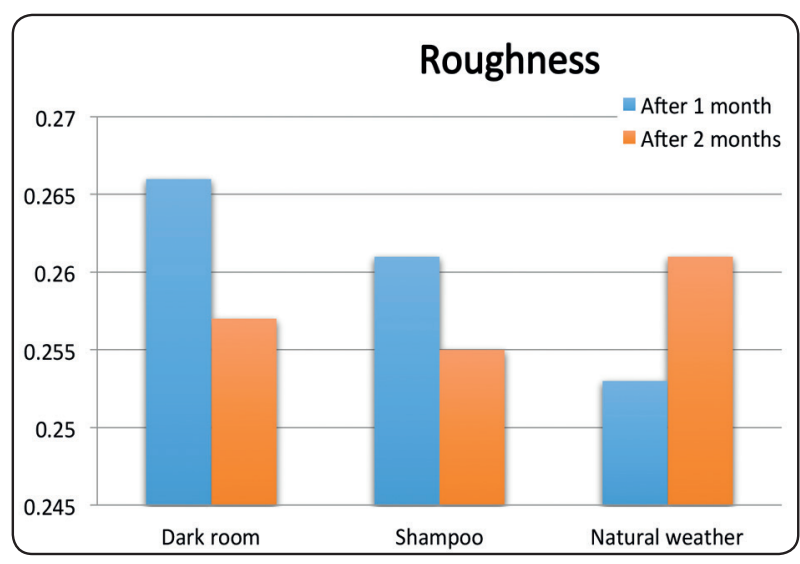

Fig (5): Bar chart represents roughness in tested groups.

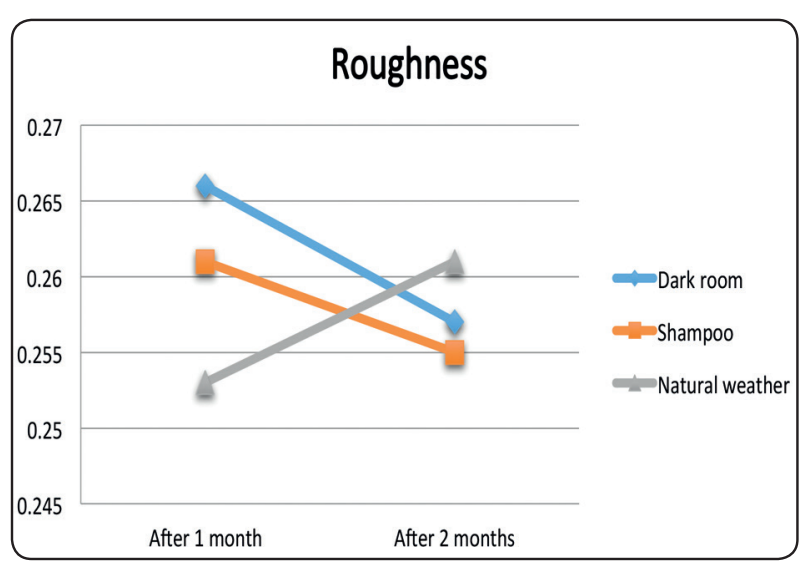

Fig (6): Line chart represents changes in roughness of tested groups during follow up 


\section{DISCUSSION}

Weather characteristics, as well as sunlight energy, temperature, moisture, dust, and pollutants disturb silicone properties. The Changes in silicone properties vary as time passes, which are expected due to alterations in structural stability of the PDMS chains as an outcome of cross-linking densities. Degradation of silicone physical and mechanical properties is usually produced by structural modifications in the distribution of the polymer molecular masses caused by polymer chain scission making the silicone softer, on the other hand enhancement of the silicone physical properties is due to intensified cross-linking, or enlarged density producing the polymer to be harder. ${ }^{6}$

Different maxillofacial silicon elastomers vary in their mechanical properties due to differences in the compositional chemical preparations; including chain length of the polymers, type, amount and size of fillers, grade of cross-linking as well as molecular weight which is responsible about changing the material from a liquid to a rubber during handling. ${ }^{11}$

Cross linking leads to tougher materials. Further, when chain scission is the dominant mechanism, the fracturing bonds inside the main chain or among them causes reduction in density of the structural network and the materials convert softer. Both mechanisms took place in the current study, and consequently in order to explore which of them dominates, mechanical and thermal analyses on silicones were done..$^{12,13}$

Regarding flexibility properties. Cross linking among the long polymer chains result in a strong inter-linking among the components, therefore, cross-linking is essential to optimize the silicon strength and provide it with high resistance to heat and degradation throughout exposure to ultraviolet light. Variation in cross-linker /elastomer ratio affects the mechanical properties of maxillofacial silicone elastomer, where it results in initial increase in mechanical properties which could be due to the formation of more cross-links and increasing crosslink density. After reaching a peak value, as in conditions such as dark room and shampoo groups, no further cross-linking sites are accessible, and the remaining un-reacted cross-linker residues in the silicone, resulting in a decrease in mechanical properties with increasing cross-linker concentration. Thus, increasing the cross-linker/ elastomer ratio causes a decline in the tensile strength of the elastomer reducing the flexibility and more brittle specimens are produced. ${ }^{11}$ Accordingly this is seen in table 1 the dark room and shampoo groups.

Exposure to sunlight has an undesirable effect on the structural stability of silicone elastomer, where UV radiation results in molecular alteration and increases the material's inherent crystallinity. This may be explained by the creation of new extremely flexible chain segments tending to reorganize themselves into higher-order formations, causing a chemicrystallization phenomenon, which is also in agreement of our study that clarify the significant increase of flexibility in natural weathering group . Table (1) ${ }^{6,15,16,17,19}$

In the meantime regarding recovery percentage incorporation of surface treated silica fillers into the base polymer permits the polymer chains to uncoil and slide past neighboring filler particles. this rises the cross-linking among neighboring PDMS chains and offers the elastomer with a advanced strength under deformation. ${ }^{11}$ This supports the results of the existing study regarding increase in the recovery percentage in the control and the shampoo groups in our study. Table (2)

Sun light aging creates denser elastomeric structures as it improves cross-linking among chains. Silicone elastomers experience crosslinking once exposed to high-energy radiation, and the quantity of cross-linking is relative to the dose and duration. Sun light aging prone to increase cross-linking, but too high levels of cross-linking 
consequence in inelastic brittle materials that ruptured at very low deformations. ${ }^{18,20}$ This explains the significant decrease in recovery percentage of natural weathering group. Table 2

Surface roughness was also tested in this study as roughness is frequently a good indicator of the performance of a mechanical factor; irregularities in the surface might produce nucleation sites for cracks or corrosion. ${ }^{3}$

In the current study regarding roughness the dark room and the shampoo groups showed decreased roughness, this can be clarified by the nonstop polymerization process, which encourages more complete polymeric chain, creating the silicone surface smoother as time goes on. , $^{1,319}$

In the meantime surface roughness was significantly increased after natural weathering which may be due to small cracks and some irregularities on the surface with reduction in the hydrophobicity by the effect of the weather conditions or may be due to high filler concentration in the material composition, which promoted a higher roughness. ${ }^{1,3,19}$ table 3

\section{CONCLUSIONS}

Regarding flexibility, there is significant increase in flexibility in natural weathering group and there is decrease in flexibility of dark room and shampoo groups .Meanwhile in recovery percentage there is increase in natural weathering group but decrease in both dark room and shampoo groups. Finally roughness decreases in dark room and shampoo groups on the other hand significant increase in the roughness of natural weathering group.

\section{REFERENCES}

1. Al-Dharrab, A. A., Tayel, S. B. \& Abodaya, M. H. The Effect of Different Storage Conditions on the Physical Properties of Pigmented Medical Grade I Silicone Maxillofacial Material. ISRN Dent. 2013, 1-9 (2013).
2. Reddy, Jr., Kumar, Bm., Ahila, S. \& Rajendiran, S. Materials in maxillo-facial prosthesis. J. Indian Acad. Dent. Spec. Res. 2, 1-4 (2015).

3. Babu, A. S., Manju, V. \& Gopal, V. K. Effect of chemical disinfectants and accelerated aging on maxillofacial silicone elastomers: AnIn vitroStudy. Indian journal of dental research : official publication of Indian Society for Dental Research 29, 67-73 (2018).

4. Griniari P, Polyzois G, P. T. Color and structural changes of a maxillofacial elastomer: The effects of accelerated photoaging, disinfection and type of pigments. J Appl Biomater Funct Mater 13, 87-91 (2015).

5. Polyzois G, L. K. Monitoring Shore A hardness of silicone facial elastomers: The effect of natural aging and silicone type after 1 year. J Craniofac Surg 25, 1217-21. (2014).

6. Aziz, T., Waters, M. \& Jagger, R. Analysis of the properties of silicone rubber maxillofacial prosthetic materials. J. Dent. 31, 67-74 (2003).

7. Mehta S, N. D. A spectrophotometric analysis of extraoral aging conditions on the color stability of maxillofacial silicone. J Indian Prosthodont Soc 17, 355-60 (2017).

8. Abouelatta, O. B. 3D Surface Roughness Measurement Using a Light Sectioning Vision System. Proc. World Congr. Eng. 1, (2010).

9. Horcas I, Fernandez R, Gomez JM, Colchero J, GomezHerrero J, and B. A. Review of Scientific Instruments. 78, 013705 (2007).

10. Kakaboura A, Fragouli M, Rahiotis C, et al. Evaluation of surface characteristics of dental composites using profilometry, scanning electron, atomic force microscopy and gloss-meter. J Mater Sci Mater Med 18, 155-63 (2007).

11. SM, Z. EC DENTAL SCIENCE Literature Review Mechanical Properties of Maxillofacial Silicone Elastomer and Approaches for their Improvement: A Literature Review. EC Dent. Sci. 8, 1293-1303 (2018)

12. Eleni, P. N., Krokida, M. K. \& Polyzois, G. L. The effect of artificial accelerated weathering on the mechanical properties of maxillofacial polymers PDMS and CPE. Biomed. Mater. 4, (2009).

13. Eleni PN, et al. Effects of outdoor weathering on facial prosthetic elastomers. Odontol. 99.1 68-76 (2011).

14. Cevik P and Eraslan OJ. Effects of the addition of titanium dioxide and silaned silica nanoparticles on the mechanical 
properties of maxillofacial silicones. J. Prosthodont. 26.7 611-615 (2017).

15. Eleni PN, et al. Structural damages of maxillofcial biopolymers under solar aging. J. Mater. Sci. Med. 18.9 1675-1681 (2007).

16. Mouzakis DE, et al. Dynamic mechanical properties of a maxillofacial silicone elastomer incorporating a zno additives:The effect of artificial aging. Craniofacial Surg. 21.6 1867-1871 (2010).

17. Eleni PN, et al. color stability of facial silicone prosthetic elastomers after artificial weathering. Dent. Res. J. 5.2 71-79 (2009).
18. Hatamleh, M. M., Polyzois, G. L., Silikas, N. \& Watts, D. C. Effect of Extraoral Aging Conditions on Mechanical Properties of Maxillofacial Silicone Elastomer. 20, 439446 (2011).

19. Goiato, M.C., Pesqueira,A.A., Santos, D.M.dos \& Dekon, S. F. de C. Evaluation of hardness and surface roughness of two maxillofacial silicones following disinfection. Braz. Oral Res. 23, 49-53 (2009).

20. Fouad M, M. M. Effect of Disinfection on Some Properties of Heat Vulcanized Maxillofacial Silicone Elastomer Reinforced by Nano Silicone Dioxide. J Bagh Coll Dent. 28, 16-21 (2016). 